\title{
Angiogenic and innate immune responses triggered by PRP in tendon cells are not modified by hyperuricemia
}

Isabel Andia

Eva Rubio-Azpeitia

Regenerative Medicine Laboratory, BioCruces, Health Research Institute, Cruces University Hospital Barakaldo, Spain

Corresponding author:

Isabel Andia

Regenerative Medicine Laboratory

BioCruces, Health Research Institute

Cruces University Hospital

P.za Cruces S/N

48903 Barakaldo, Spain

E-mail: iandia2010@hotmail.com

\section{Summary}

Background: hyperuricemia is becoming a critical medical problem, and a current focus of research. Uric acid is also a death cell associated stressor that may trigger innate immune responses via the synthesis of inflammatory and angiogenic proteins. Purpose: to investigate the angiogenic/inflammatory protein profile of tendon cells treated with Platelet Rich Plasma (PRP), and to assess if there are any differences in synthesis of angiogenic/inflammatory cytokines between PRP-treated or hyperuricemic PRP-treated cells. Methods: tendon cells were treated with PRP or hyperuricemic PRP and cell culture supernatants examined using glass based protein arrays for inflammation and angiogenesis. Relevant proteins were subsequently quantified by ELISA or EASIA methods. Results: the impact of PRP on angiogenesis and inflammation is evidenced by relevant cytokine synthesis including: Monocyte Chemoattractant Protein (MCP-1/CCL2), Regulated upon Activation Normally $T$ cells Expressed and Secreted (RANTES/CCL5), IL-6/CXCL6, IL-8/CXCL8, Vascular Endothelial Growth Factor (VEGF), Growth Regulated Protein (GRO-a/CXCL1) and Hepatocyte Growth Factor (HGF). IL-1beta was not detected in these conditions. Taken together these data suggest an initial angiogenetic and innate immune responses driven by chemokines that is not altered by the presence of hyperuricemia, at this point, except for IL-8 secretion, $p=0.042$.
KEY WORDS: PRP, uric acid, tenocyte, chemokines, growth factors.

\section{Introduction}

Hyperuricemia, high levels of uric acid in body fluids, is becoming a critical medical problem ${ }^{1}$. Its prevalence and its related comorbidities have increased dramatically in the last decades, and its significance, previously underestimated, is currently a focus of research ${ }^{2}$. In fact, gout (urate overload disease) is an expensive disease, and the number of gout sufferers in the US is more than double the patients with rheumatoid arthritis ${ }^{1}$.

The possibility that high levels of uric acid in biological fluids play a role in the development and progression of tendinopathy is based on recent knowledge on the influence of MSU crystals in tendon biology ${ }^{3}$. This is supported by the notion of uric acid acts as a death cell associated stressor, a danger signal (DAMP) that may stimulate macrophages to produce proinflammatory mediators such as $\mathrm{IL}-1 \mathrm{~b}^{4,5}$.

However, hyperuricemia is a nondramatic alteration of tissue homeostasis, in contrast to the more substantial alteration provoked when uric acid condensates, and forms monosodium urate (MSU) crystals, the hallmark of gout. Recently, we have shown that high concentrations of uric acid in the tendon cell microenvironment involve a mild alteration in extracellular matrix homeostasis in the context of platelet rich plasma (PRP) ${ }^{6}$.

$\mathrm{PRP}$ is a plasma preparation, obtained from autologous peripheral blood that contains a platelet level above peripheral blood and, as often found in tendon treatment, it can hold a high concentration of leukocytes (L-PRP) $)^{7,8}$. In the last years, the use of PRPs has expanded to meet multiple medical problems where current treatment options are judged to be suboptimal ${ }^{9}$. For example, PRP is used as an injectable to treat tendinopathy with moderate clinical success in diverse tendons ${ }^{10}$. When injected within the tendons, PRP release a molecular pool that targets specialized tendon fibroblasts triggering the synthesis of at least two different groups of functional proteins: first, proteins that regulate the architecture and homeostasis of ECM and, secondly cytokines, growth factors and chemokines that act in a paracrine manner inducing a low-grade inflammation associated to angiogenesis ${ }^{11,12}$.Thus, PRP is also a valuable research tool to study cell behavior when exposed to a multimolecular environment in a physiological context. 
Several studies have suggested that angiogenic and inflammatory modulators may play a role during the progression of tendinopathic conditions seeing that they are present in painful and ruptured tendons ${ }^{13}$. Furthermore, recent data show that patients with metabolic diseases are prone to develop tendinopathies ${ }^{14}$.

In this study we explore first the response of tendon cells to the PRP-released molecular pool in terms of the synthesis of angiogenic and inflammatory modulatory proteins. Secondly, in parallel, we perform an exploratory study to assess whether hyperuricemia can be sensed as stressor by tenocytes, and whether this stressor can modify the angiogenic/inflammatory response to PRP.

\section{Materials and methods}

\section{Tendon cell culture and cell treatments}

Tendon cells were obtained through explant culture from the semitendinosus tendon of two healthy young donors undergoing ACL surgery, after informed consent and local ethic committee approval. Briefly, samples were minced and incubated at $37^{\circ} \mathrm{C}, 5 \% \mathrm{CO}_{2}$. After cell adhesion in the plastic, tissue fragments were retired carefully, and cells allowed grow until confluence. Then, cells passaged to a T75 flask at a density of 4000 cells $/ \mathrm{cm}^{2}$, were treated in parallel with $10 \%$ PRP or $10 \%$ hyperuricemic PRP for $96 \mathrm{~h}$. We used pure PRP (no leukocytes) with a moderated enrichment of platelets [2.30 (S.D. $=0.68$ ) fold above peripheral blood] obtained from four donors (2 men and 2 women). This PRP is the same we use in our clinical studies ${ }^{15}$. The concentration of uric acid in hyperuricemic cultures was $20 \mathrm{mg} / \mathrm{dL}$.

Constitutive basal secretion of untreated cells was assessed after 30 hours starvation with $0.2 \%$ PRP in DMEM. Cell culture supernatant containing proteins secreted by tendon cells during PRP or hyperuricemic PRP treatment were harvested after $96 \mathrm{~h}$ of treatment exposure, aliquoted for protein measurements, and stored at $-20^{\circ} \mathrm{C}$ until analyses. Cell number was determined after trypsinization by means of a Bürker Chamber using the formula: Cells $/ \mathrm{ml}=$ (cells counted in 4 large squares/4) $\times 10^{4} \times$ dilution factor.

\section{Protein profiling: glass based arrays to detect in- flammatory and angiogenic cytokines}

Using RayBio ${ }^{\circledR}$ Human Angiogenesis Antibody Array G-Series 1000 Cat\# AAH-ANG-G1000-4, and RayBio® Human Inflammation Antibody Array G-Series 3 Cat\# AAH-INF-G3-4, (RayBiotech Inc Norcross GA) multiple cytokines and chemokines involved in angiogenesis and inflammation were simultaneously screened (http://www.raybiotech.com). Protein arrays comprise many different antibodies arrayed on a glass support. Each antibody is specific, and captures its target protein from the complex protein mixture contained in cell supernatants. This is followed by detection and quantification.

Briefly, cell supernatants were collected after PRP or hyperuricemic PRP treatments and pooled equally for protein screening by means of RayBio ${ }^{\circledR}$ pre-assembled antibody array glass chips. The assays were performed according to the manufacturer's instructions and protein signals normalized against positive controls representing equal amounts of biotinylated immunoglobulins printed directly onto the array. These arrays provide a semiquantitative assessment of multiple proteins but needs subsequent confirmation and quantification.

\section{Quantification of proteins by ELISA or EASIA}

Positive results in the protein antibody array were validated using commercially available solid phase sandwich enzyme linked immunosorbent assays (ELISA) for HGF (R\&D Systems, Inc, Minneapolis MN, USA) and VEGF (-165) (In vitrogen Corporation, Caramillo CA, USA), GRO-alpha and MCP-1 (RayBiotech Inc, Norcross, GA). Solid phase enzyme amplified sensitivity immunoassays (EASIA) based on oligoclonal systems are used for RANTES, IL-6 and IL- 8 determinations (Biosource Europe SA, Nivelles, Belgium).

\section{Statistical analysis}

The experiments were performed in triplicate for each of the four PRP donors per two tendon donors. The concentration of proteins is shown as median and 2575 percentiles. Spearman coefficient was used to describe correlations. $P$ values were determined using the Wilcoxon test for non-parametric matched values. A p value of less than 0.05 was considered to be significant. Data were analyzed using SPSS for windows, version 18.0, SPSS (SPSS Inc, Chicago, IL USA).

\section{Results}

Profile of angiogenic proteins secreted by tendon cells treated with PRP or hyperuricemic PRP

The first angiogenic array (AAH-ANG-G1) detected fourteen out of twenty proteins. Most abundant proteins are shown in Figure $1 \mathrm{~A}, \mathrm{~B}$. The second angiogenic array (AAH-ANG-G2) detected twenty-two out of twenty three proteins. However the intensity of the signals was low except for the proteins shown in Figure $1 \mathrm{C}$. Overall, we found detectable levels of 36 angiogenic proteins but with differing intensities.

Very abundant proteins present in cell culture supernatants were angiogenin, angiostatin, Growth Regulated Oncogene (GRO-a/CXCL1), Regulated upon Activation Normally $T$ cells Expressed and Secreted (RANTES/CCL5), IL-6/CXCL6, IL-8/CXCL8, and Monocyte Chemoattractant Protein (MCP-1/CCL2) (Fig. 1 A, C). Relevant levels of tissue inhibitors of 


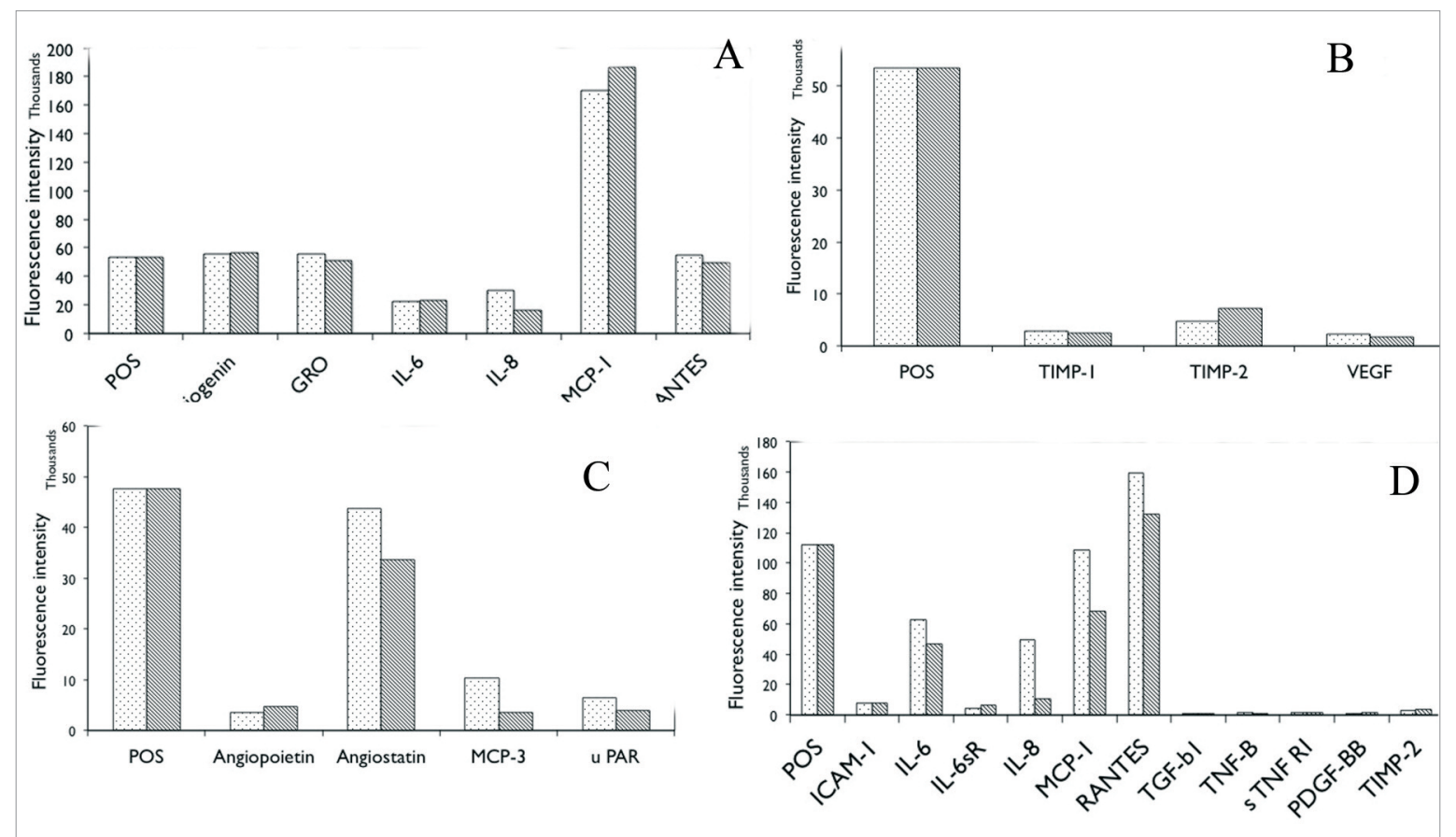

Figure 1. Main results obtained from human protein arrays (RayBio, RayBiotech) after analyzing pooled cell supernatants (from two cell donor and four PRP or hyperuricemic PRP per cell donor). Dotted bars represent supernatants from PRP treated tendon cells and stripped bars represent supernatants from tendon cells treated with hyperuricemic PRP. A and B highly and moderately abundant proteins detected by means of AAH-ANG-G1. C represents main proteins detected by means of (AAH-ANG-G2). D represents primary inflammatory proteins as assessed by means of AAH-INF-G3-4, (all arrays from RayBiotechlnc Norcross GA).

Abbreviations GRO- $\alpha / C X C L 1$, Growth Regulated Protein; HGF, Hepatocyte Growth Factor; ICAM, intercellular adhesion molecule; IL-6/CXCL6, Interleukin 6; IL6sR, soluble receptor for IL-6; IL-8/CXCL8, Interleukin 8; MCP-1/CCL2, Monocyte Chemoattractant Protein; PDGF-BB, platelet derived growth factor; POS, positive control; RANTES/CCL5, Regulated upon Activation Normally T cells Expressed and Secreted; sTNF RI, soluble receptor for TNF;TGF-b1, transforming growth factor; TIMP-1, -2, tissue inhibitor of metalloproteinase; TNF-B, tumor necrosis factor beta; VEGF, Vascular Endothelial Growth Factor.

metalloproteinases, TIMP-1, TIMP-2, MCP-3, angiopoietin, Vascular Endothelial Growth Factor (VEGF), and UPAR were also evidenced (Fig. $1 \mathrm{~B}, \mathrm{C}$ ).

Profile of angiogenic proteins secreted by tendon cells treated with PRP or hyperuricemic PRP

Because inflammation and angiogenesis often occur in parallel several angiogenic proteins are also included in the inflammatory array. Therefore we were able to corroborate elevated levels of MCP-1, RANTES, IL- 6 and IL-8 in a different array. Additionally, ICAM, sIL-6R, TGF-b1, TNF-B, sTNF R1 and PDGF-BB were detected with a moderate intensity in the inflammatory array.

Quantitative assessment of inflammatory and angiogenic proteins secreted by tendon cells after PRP and hypeuricemic PRP treatment

Chemokines can be homeostatic, inducible or both. Our data show that tendon cells constitutively secrete low levels of VEGF, and IL8 but moderate levels of HGF, MCP-1 and GRO- $\alpha$ (Tab. 1). The secretion of chemokines (RANTES, MCP-1, IL-6 and IL-8) and VEGF and HGF is boosted by PRP treatment. There were no differences between PRP or hyperuricemic PRP, except for IL-8 that showed a significant decrease in the presence of hyperuricemia, $p=0.042$, (Fig. 2). VEGF and GRO-a showed an increase in the presence of hyperuricemia, although not statistically significant.

PRP induces a para-inflammatory response marked by the absence of relevant pro-inflammatory cytokines such as IL-1beta but characterized by elevated synthesis of chemokines inducing leukocyte migration. Most abundant secreted angiogenic/inflammatory chemokines in response to PRP are involved in monocyte/macrophage attraction, including MCP1, RANTES. Additionally we have evidenced a relevant synthesis of $\mathrm{GRO}-\alpha$, also named neutrophil-activating protein, in parallel with the synthesis of IL-8 known to be chemoattractant for neutrophils. Tendon cells produce a relevant amount of HGF, a crucial antiinflammatory protein in the context of PRP (Fig. 2A). 
Table 1. Constitutive chemokine and growth factor secretion by tendon cells and fold increase after 96h PRP treatment or hyperuricemic PRP treatment.

\begin{tabular}{|c|c|c|c|}
\hline & $\begin{array}{l}\text { Constitutive secretion } \\
\text { (ng/million cells) } \\
\text { Mean (SD) }\end{array}$ & $\begin{array}{l}\text { PRP (FOLD increase } \\
\text { over constitutive) } \\
\text { median (min.-max.) }\end{array}$ & $\begin{array}{l}\text { Hyperuricemic PRP } \\
\text { (FOLD increase over constitutive) } \\
\text { median (min.-max.) }\end{array}$ \\
\hline HGF & $7.22(1.53)$ & $\begin{array}{l}6.00 x \\
4.71-6.67\end{array}$ & $\begin{array}{l}4.62 x \\
3.57-7.34\end{array}$ \\
\hline VEGF & $0.49(0,23)$ & $\begin{array}{l}6.91 x \\
5.57-10.56\end{array}$ & $\begin{array}{l}9.56 x \\
7.28-10.69\end{array}$ \\
\hline MCP-1/CCL2 & $5.76(1.13)$ & $\begin{array}{l}6.67 x \\
3.53-7.78\end{array}$ & $\begin{array}{l}5.33 x \\
2.80-7.24\end{array}$ \\
\hline RANTES/CCL5 & $3.0(0.73)$ & $\begin{array}{l}\text { 42.32x } \\
24.6-53.9\end{array}$ & $\begin{array}{l}\text { 48.02x } \\
29.2-76.2\end{array}$ \\
\hline IL-6/CXCL6 & $2.18(0.73)$ & $\begin{array}{l}9.35 x \\
8.11-10.74\end{array}$ & $\begin{array}{l}9.10 x \\
4.57-10.93\end{array}$ \\
\hline IL-8/CXCL8* & $0.73(0.10)$ & $\begin{array}{l}19.75 x \\
13,61-23,99\end{array}$ & $\begin{array}{l}6.61 x \\
1,27-9,61\end{array}$ \\
\hline GRO-a/CXCL2 & $19.0(1.48)$ & $\begin{array}{l}2.84 x \\
0.4-5.2\end{array}$ & $\begin{array}{l}3.58 x \\
3.4-5.4\end{array}$ \\
\hline
\end{tabular}

${ }^{*} p=0.042$

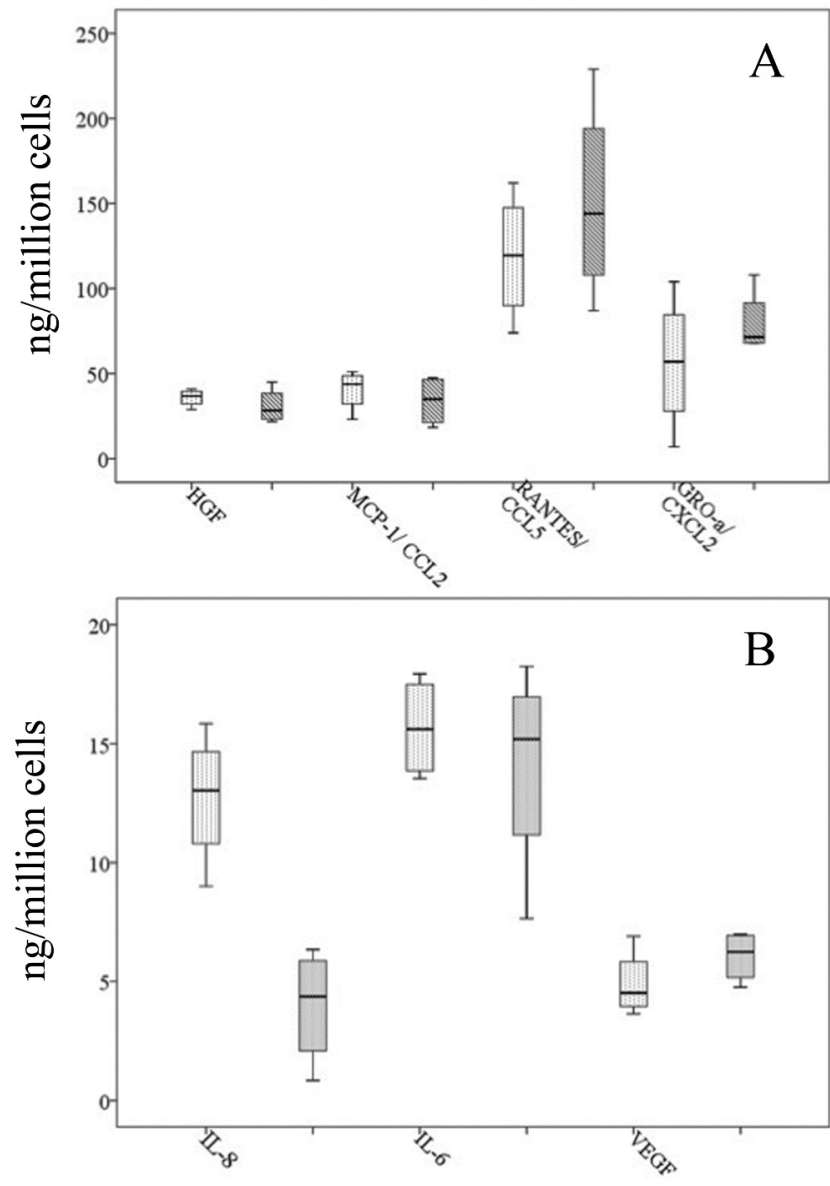

Figure 2. Quantification of selected chemokines and growth factors by ELISA and EASIA. Comparison of angiogenic and inflammatory proteins in PRP (dotted bars), and hyperuricemic PRP. treated cells (striped bars). Box plots represent median values and $25^{\text {th }}$ and $75^{\text {th }}$ percentiles. A, RANTES, MCP-1, GRO- $\alpha$, and HGF. B, VEGF, IL-8 and IL-6. There were no significant differences between groups, except for a significant decrease in the synthesis of IL-8, $p=0.042$. 


\section{Discussion}

Technological advances in the past decade have changed the way experiments are performed; to obtain a global view of the synthesis of inflammatory and angiogenic proteins by PRP-treated tendon cells, we have performed several protein arrays and we have examined angiogenetic paracrine signaling and inflammation. What emerges from these semi-quantitative results is that tenocytes are a source of numerous chemokines, extracellular signaling factors of the response to different stressors. We also found that, in this in vitro model, hyperuricemia is a minor stressor for tendon cells that does not modify significantly the angiogenic or para-inflammatory responses induced by PRP. In fact, major inflammatory triggers such as IL-1beta are not induced by PRP or hyperuricemic PRP.

In contrast, we found relevant synthesis of chemokines, chemotactic cytokines with the ability to guide the migration of immune cells. Importantly, we proof that tendon cells synthesize relevant amounts of monocyte chemoattractant protein (MCP-1/CCL2) and RANTES/CCL5. Both MCP-1 and RANTES mediate migration of monocyte/macrophages and are involved in inflammatory and angiogenetic mechanisms. These chemokines are typically induced during an innate immune response, and may also have a role as homeostatic chemokines involved in normal processes of tissue maintenance. The role of these chemokines in the healing tendon has been the focus of recent research. Importantly, both CCL5 and CCL2 were expressed in the healing proper tendons in a rat model with subsequent macrophage infiltration ${ }^{16}$. Moreover, the expression of chemokines was shown to precede the growth of nerve fibers in the Achilles tendon.

Although our results need in vivo confirmation it becomes apparent that a possible mechanism behind PRP is the enhancement and acceleration of the activation of the innate immune response by local tendon cells. Thus PRP therapies may be especially relevant in tendinopathic conditions marked by a failed healing response.

The production of these chemokines is similar in the presence of hyperuricemia. Of note, hyperuricemia elevates circulating CCL2 (MCP1) levels and primes monocyte trafficking in subjects with intercritical gout ${ }^{17}$ and serum MCP-1 is also elevated in patients with hyperuricemia compared to normouricemic controls. A possible source of increased serum MCP-1 includes not only circulating monocytes and macrophages but also local cells such as tenocytes. Notwithstanding in our cell culture model, hyperuricemia is a minor stressor for tenocytes that does not induce changes in MCP-1 but in the synthesis of IL-8 confirming a previous study6.

However, we cannot rule out that hyperuricemia could modify the polarization of infiltrating monocytes/macrophages ${ }^{18}$. Macrophages are involved in maintaining the inflammatory state (functional consequences of innate immune responses), or resolving it ${ }^{19}$. To do so they polarized into different molecular states depending on the local signals of the environment. Thus, further research is needed to assess the polarization state of macrophages in the presence of PRP and hyperuricemic PRP in tenocyte co-cultures. Regarding inflammation, we did not detect production of IL-1b. Nevertheless, we detected a minimal intensity for IL-1alpha and TNF-alpha indicating that tenocytes might be marginally inflamed, but the presence of IL-1alpha and TNF-alpha coexists with slight levels of IL-10, which dampens inflammation. Even so, the presence of IL-6, IL-8 and GRO-a may indicate a parainflammatory state. Importantly, tenocytes synthesize relevant amounts of HGF, a major player in the anti-inflammatory mechanism of PRP in tendon cells ${ }^{20}$.

Regarding angiogenesis, previous research has shown that VEGF and HGF are secreted by tendon cells exposed to PRP treatment ${ }^{21,22}$. The former is a well-known angiogenic factor targeting endothelial cells and stimulating their proliferation. HGF is a pleiotropic factor involved in cell motility and the formation of tubes in angiogenesis.

What arises from the current data is production of several angiogenic proteins (HGF, VEGF angiogenin, angiopoietin, IL-8, MCP-1 and RANTES) by tendon cells as a rapid response to PRP treatment. Present work also extends previous findings since we also evidence an important concentration of other relevant proteins in angiogenesis including angiogenin and angiopoietin. The former is a potent stimulator of new blood vessel formation that exerts its activity by binding to actin in the surface of endothelial cells being subsequently endocytosed and translocated to the nucleus. Angiogenin is also involved in degradation of the basement membrane allowing endothelial cells penetration into the tendon. The latter mediates reciprocal interactions between the endothelium, surrounding matrix and mesenchyme.

To our knowledge, for the first time, it is shown that PRP treated tenocytes are a relevant source of angiogenin, IL-8 and GRO-a, chemokines known to mediate chemotaxis and activation of neutrophils as well as angiogenesis. We also show for the first time, that signal transduction of PRP released signaling cytokines is reflected in an increase of pro-angiogenic proteins and a para-inflammatory response mainly involving monocyte/macrophage chemotaxis.

One limitation of our study is that we did not assess sIL-6R that regulates the IL-6 trans-signaling ${ }^{23}$. In fact while trans-signaling is pro-inflammatory, classic IL-6 signaling via IL-6R membrane receptor is necessary for the anti-inflammatory actions of the cytokine. Moreover, cell culture experiments are of limited value because they do not reflect the complex interactions between the multiple cell phenotypes involved in tissue healing and pathology. Positive and negative feedback loops, pleiotropic effects and redundant functions, spatial and temporal expression of or synergistic interactions between multiple cytokines, even regulation via release of soluble forms of membranebound receptors, all are common mechanisms modulating the effects of cytokine signaling. 
However, taken together our data are provocative, and suggest an innate immune response driven by monocytes/macrophages in response to PRP administration. Manipulating angiogenesis and inflammation by means of PRP administration is a relevant tool for tissue regeneration. There is hope that a better understanding of angiogenic and para-inflammatory mechanisms will help in refining PRP formulations for tendinopathies. Further research is warranted ${ }^{24}$.

\section{Acknowledgement}

The study was supported by SAIO2012-PE12BF007. We thank Eric Mennesson for technical assistance with protein arrays.

The authors declare no conflicts of interest.

\section{References}

1. Crittenden DB, Pillinger MH. The year in gout. 2011-2012. Bull NY Hospital Joint Diseases. 2012;70(3):145-151.

2. Richette P, Bardin T. Gout. The Lancet. 2010;375:318-328.

3. Chana A, Callon KE, Dray M, et al. Interactions between tenocytes and monosodium urate monohydrate crystals: implications for tendon involvement in gout. Ann Rheum Dis. 2014.

4. Martinon F. Update on biology: uric acid and the activation of immune and inflammatory cells. Curr Rheumatol Rep. 2010;12(2):135-141.

5. Rock KL, Kataoka H, Lai J-J. Uric acid as a danger signal in gout and its comorbidities. Nat Rev Rheumatol. 2013; 9(1):1323.

6. Andia I, Rubio-Azpeitia E, Maffulli N. Hyperuricemic PRP in tendons. Biomed Res Int. 2014.

7. DohanEhrenfest DM, Andia I, Zumstein MA, Zhang CQ, Pinto $\mathrm{NR}$, BieleckiT. Classification of platelet concentrates (PlateletRich Plasma-PRP, Platelet-Rich Fibrin-PRF) for topical and infiltrative use in orthopedic and sports medicine: current consensus, clinical implications and perspectives. Muscles Ligaments Tendons J. 2014;4(1):3-9.

8. Andia I, Maffulli N. Platelet-rich plasma for muscle injury and tendinopathy. Sports Med Arthrosc. 2013;21(4):191-198.

9. Andia I, Abate M. Platelet-rich plasma: underlying biology and clinical correlates. Regen Med. 2013;8(5):645-658.

10. Andia I, Latorre PM, Gomez MC, Burgos-Alonso N, Abate M, Maffulli N. Platelet-rich plasma in the conservative treatment of painful tendinopathy: a systematic review and meta-analysis of controlled studies. Br Med Bull. 2014;110(1):99-115.

11. Andia I, Sánchez M, Maffulli N. Joint pathology and plateletrich plasma therapies. Expert Opin Biol Ther. 2012;12(1):72211.

12. Andia I, Maffulli N. Platelet-rich plasma for managing pain and inflammation in osteoarthritis. Nat Rev Rheumatol. 2013 9(12):721-730

13. Ackermann PW, Franklin SL, Dean BJ, Carr AJ, Salo PT, Hart DA. Neuronal pathways in tendon healing and tendinopathy update. Front Biosci (Landmark Ed). 2014;19:1251-1278.

14. Abate M, Schiavone C, Salini V, Andia I. Occurrence of tendon pathologies in metabolic disorders. Rheumatology (Oxford). 2013;52(4):599-608.

15. Martin JI, Merino J, Areizaga LM, Gomez-Fernandez MC, Burgos-Alonso N, Andia I. Platelet-rich plasma (PRP) in chronic epicondylitis: study protocol for a randomized controlled trial. Trials. 2013;14:410.

16. Stålman $A$, Bring $D$, Ackermann PW. Chemokine expression of CCL2, CCL3, CCL5 and CXCL10 during early inflammatory tendon healing precedes nerve regeneration: an immunohistochemical study in the rat. Knee Surg Sports Traumatol Arthrosc. 2014

17. Grainger R, McLaughlin RJ, Harrison AA, Harper JL. Hyperuricaemia elevates circulating CCL2 levels and primes monocyte trafficking in subjects with inter-critical gout. Rheumatology (Oxford). 2013;52(6):1018-1021.

18. Uratsuji H, Tada $Y$, Kawashima T, et al. P2Y6 receptor signaling pathway mediates inflammatory responses induced by monosodium urate crystals. J Immuno. 2012;188(1):436-444.

19. Chen GY, Nunez G. Sterile inflammation: sensing and reacting to damage. Nat Rev Rheumatol. 2010;10:826-837.

20. Zhang J, Middleton KK, Fu FH, Im H-J, Wang J H-C. HGF mediates the anti-inflammatory effects of PRP on injured tendons. PLOS ONE. 2013;8(6)e67303.

21. Anitua E, Andia I, Sanchez M et al. Autologous preparations rich in growth factors promote proliferation and induce VEGF and HGF production by human tendon cells in culture. J Orthop Res. 2005;23(2):281-286.

22. Anitua E, Sanchez M, Nurden AT, et al. Autologous fibrin matrices: a potential source of biological mediators that modulate tendon cell activities. J Biomed Mater Res A. 2006;77(2):285293.

23. Rose-John S, Waetzig GH, Scheller J, Grötzinger J, Seegert D. The IL-6/s IL-6R complex as a novel target for therapeutic approaches. Expert OpinTher Targets. 2007;11(5):613-624.

24. Padulo J, Oliva F, Frizziero A, Maffulli N. Muscles, Ligaments and Tendons Journal. Basic principles and recommendations in clinical and field science research. MLTJ. 2013;4:250-252. 